\title{
A local search algorithm to allocate loads predicted by spatial load forecasting studies
}

\author{
Joel D. Melo ${ }^{\mathrm{a}, *}$, Sergio Zambrano-Asanza ${ }^{\mathrm{b}}$, Antonio Padilha-Feltrin ${ }^{\mathrm{c}}$ \\ a The Engineering, Modeling and Applied Social Sciences Center, Federal University of ABC-UFABC, Santo André, SP, Brazil \\ b Planning Department, the CENTROSUR Distribution Utility, Cuenca, Ecuador

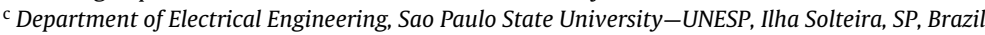

\section{A R T I C L E I N F O}

\section{Article history:}

Received 9 August 2016

Received in revised form 12 January 2017

Accepted 13 January 2017

Available online 7 February 2017

\section{Keywords:}

Power distribution system planning

Geographic information system

Shortest-path method

Spatial load forecasting

\begin{abstract}
A B S T R A C T
In recent years, spatial load forecasting studies have helped to direct the expansion of the distribution systems in cities with rapid urban growth, providing maps that showing the spatial distribution of expected load. However, these maps do not allow to determine how load varies on the existing network elements. This information is important to define the reinforcements or the installation of new facilities in the electrical distribution network. In order to help planners in such decisions, a search method to allocate the loads resulting from spatial load forecasting studies is presented. This method treats each of these forecast loads as new load center to be connected to an existing distribution feeder. To find the path from a load center, the proposed method uses a list of its nearby feeders. Allocation depends on the path cost function, which is calculated based on the supply capability of the network elements. The proposal chooses the shortest path with sufficient capacity to supply the new load, i.e., it finds the path with minimal cost function for list of nearby feeders. The result is the final available capability of existing networks (after the allocation process) to supply the expected loads in the geographic area. The method is tested using the results of a spatial load forecast for a real distribution system in a medium-sized Brazilian city. In this test system, the load allocation influenced the number of network elements to be reinforced. The proposal was compared to commercial software, showing a configuration with smaller numbers of overload elements and a lower cost of expansion to the most overloaded feeders.
\end{abstract}

(c) 2017 Elsevier B.V. All rights reserved.

\section{Introduction}

A geographic information system (GIS) helps utility planners visualize and analyze georeferenced data in order to obtain information useful for making decisions regarding expanding their business [1]. In the power distribution planning process, a GIS allows planners to understand load-growth relationships, patterns, and trends $[2,3]$. The implementation of smart grids has encouraged the use of GIS by distribution utilities because it supports business management of distribution networks [4,5].

Rapid urban growth in developing countries and urban redevelopment result in new challenges for the planners of power networks when deciding how to expand distribution systems. Planners must perform spatial electric load forecasting studies before expanding distribution systems in urban zones [6].

\footnotetext{
* Corresponding author.

E-mail addresses: joel.melo@ufabc.edu.br, joeldavidm@gmail.com (J.D. Melo).
}

Spatial electric load forecasting methods divide the city into several subareas in order to estimate load growth, displaying the expected spatial load distribution on thematic maps [7].

\subsection{Motivation}

The thematic maps resulting from spatial load forecasting methods are extremely valuable for decisions regarding investments needed to expand power networks. In order for these maps to aid distribution system design, the forecast loads must be allocated to distribution network elements [8]. This allocation could supply useful information for making decisions, identifying the capability shortfalls of electrical network elements and the location of new facilities. Also, this information could help the planners in the creation of a suitable load database for optimal expansion algorithms of the distribution system, reducing the search space of the network elements to be expanded, as explained in Refs. [9,10].

Load allocation could be performed using GIS software applications $[8,10]$ and allocation-location models [11], which are used by other class of public service companies for allocating infrastructure 
to meet the demands of its users. For example, installation of public health centers [12] and public schools [13]. However, these models cannot be applied to distribution utilities for allocating loads because they do not take into account distribution network capacity constraints.

In this paper, load allocation is the main goal and is calculated using a local search algorithm [14,15] given the locations of new load centers to be connected to a distribution feeder. Each load center is a point at which the load of a subarea is assumed to be concentrated.

\subsection{Contributions}

This paper presents a method that allocates a forecast load to distribution feeders, seeking to reduce the number of overloaded existing network elements (after the allocation process). The main contributions of the proposed method are explained below.

a) Reduce the number of overloaded existing network elements: the proposal considers the variation in network element supply capability during the load allocation process. The allocationlocation models available in GIS software do not take into account this variation. At each step of the proposed load allocation, the capabilities of the network elements are updated, so when the proposal seeks to allocate the next load center, will find a new path with the highest available capacity. This allocation process reduces the number of overloaded existing network elements, which could help in making investments decisions because of the lead time for adding new facilities in the distribution systems.

b) Reduce the search space of the network elements to be expanded: the proposed method finds solutions with fewer overloaded existing network elements than similar methods available in GIS software. The performed minimization the number of overloaded feeders by the proposed method could help to the planners in the creation of a suitable load database for optimal expansion algorithms of the distribution system, reducing the search space of the network elements to be expanded.

c) The computational time needed is similar to that of specialized software: Dijkstra's algorithm available in GIS has the lower computational cost compared to other local search algorithms. The proposed method and Dijkstra algorithm are similar in terms of computational time. However, the proposed method can work with positive and negative values of the path's cost function, being this a differential of the proposal in comparison of search algorithms that consider these values, but with a higher computational effort.

\subsection{Organization of the paper}

This paper is organized as follows. In Section 2, it has been discussed how to allocate the forecast load from spatial load forecasting studies in electrical distribution networks. In Section 3, an overview of search algorithms is explained in order to show the differences with the proposed method. In Section 4, the proposed methodology is presented, explaining the input data for implementation the proposal (Section 4.1), and how the list feeders and load centers can be constructed in order to determine the space search of the proposed methodology (Section 4.2). The main differential of the proposal compared with the search algorithms available in GIS is the path's cost function, which is explained in Section 4.4. The steps for the proposed load allocation are explained in detail using an example with two feeders and a load center are presented in Sections 4.4. In Section 5, the proposed methodology by comparing the ArcGIS software provided with real data from a Brazilian city is applied to allocate the forecast load. The concluding remarks are presented in Section 6.

\section{Allocation of forecast loads in a distribution network}

The allocation of forecast loads to elements in a distribution network based on their spatial distribution is not addressed by the methodologies available in the specialized literature on this kind of forecasting.

Linear and non-linear programming algorithms may be used to allocate predicted loads resulting from grid-based spatial load forecasting simulations to network elements [16], but their computing time increases considerably with grid size, hindering their use for large distribution systems.

GIS software applications could also be used to allocate new loads. For example, a Voronoi diagram can be used to determine the feeder boundaries for the allocation of forecast loads to distribution feeders [17]. However, a diagram of this type does not take into consideration the constraints of the capacities of distribution networks.

Local search algorithms have low computing time even when there is a large quantity of input data [8], making them suitable for load allocation in distribution networks. In order to implement them, a graph defined by a set of vertices and edges is employed, and a path (connection) between the new load and a substation is sought such that the path's cost function is the minimum of a list of candidates. This function is equal to the sum of the costs of each edge traversed through the graph, from a new load to a substation [18].

Allocation-location models available in GIS software employ Dijkstra's algorithm, or some variation thereof, for a local search in order to perform load allocation because of the lower computational cost compared to other local search algorithms [19,11]. Dijkstra's method requires that the cost of each edge be greater than zero in order to find the shortest path for a new load. This does not permit the use of network element supply capabilities and their variation during the allocation process as edge costs because the value of the capability could be negative.

To assist a distribution utility in making decisions on how to expand its distribution system, we propose a local search algorithm that calculates the cost function based on the variation of the available capability of the existing distribution networks to supply the expected loads, seeking to reduce the number of overloaded existing network elements (after allocation process). This is the main difference between our method and similar methods found in the specialized literature.

\section{Search algorithms}

The allocation of new load can be approached as an application in the field of network analysis for the calculation of shortest paths. A very common algorithm available in GIS for calculating the shortest distance between two vertices is the Dijkstra algorithm. It is used in edge-weighted graphs. Using the Dijkstra algorithm, it is possible to determine the shortest path between a new load center (vertex) and any other point in the electrical network (an existing vertex in graph). In the allocation process, this algorithm calculates the shortest distance beginning from a starting point (the location of the new load center) continuously, and to exclude longer distances when making an update. It consists of the following steps:

Step 1-Initialize all vertices (connection points on the electrical network) with distance equal to bigger number ("infinite") from a new load center.

Step 2-Mark the distance from a location of new load center as permanent, and all other distances as temporarily. 


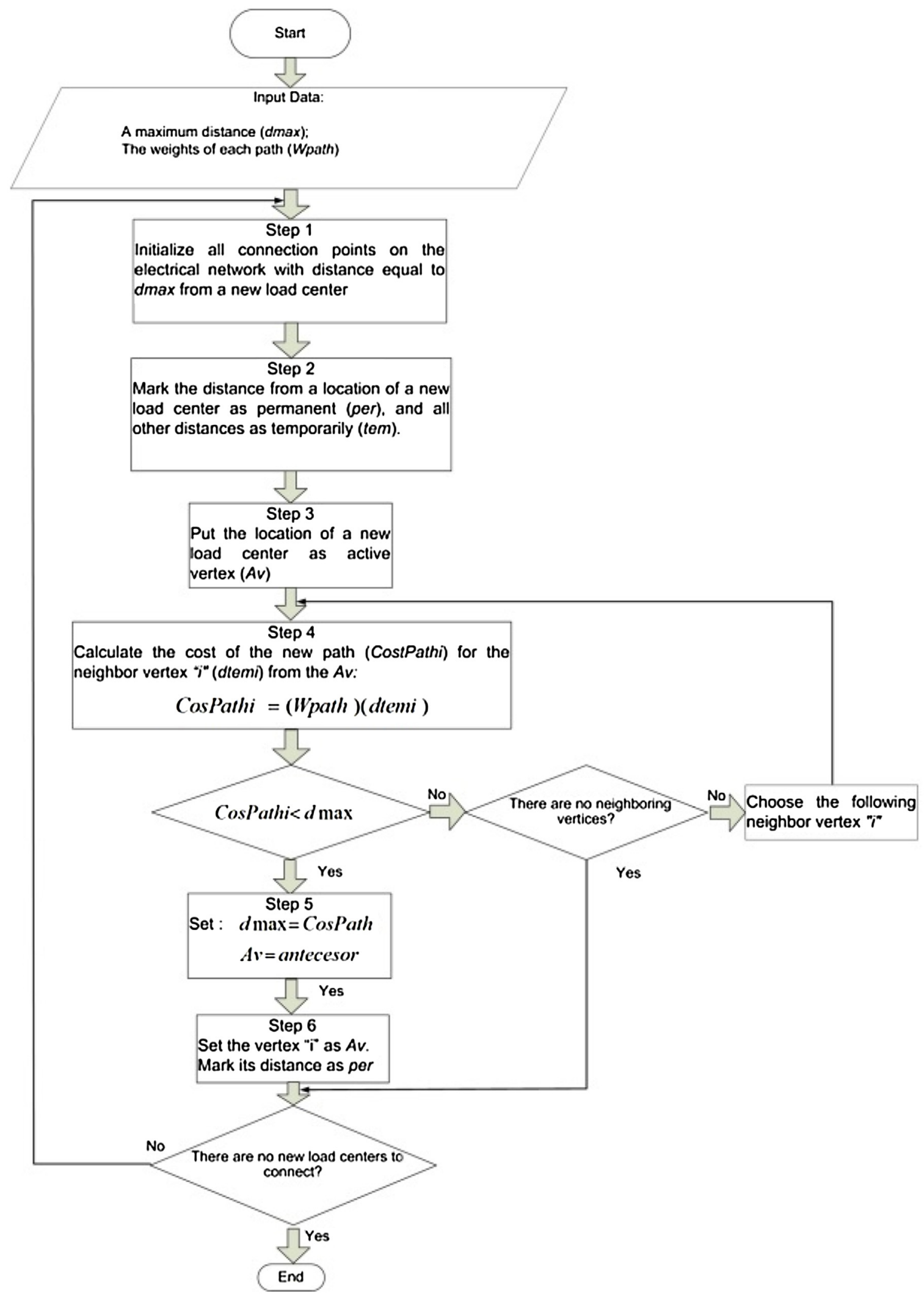

Fig. 1. Flowchart of the Dijkstra algorithm.

Step 3-Put the location of a new load center as active vertex. Step 4-Calculate the temporary distances of all neighbor vertices from the active vertex by summing up its distance with the weights of the edges (cost of the new path).
Step 5-If such a calculated distance of a vertex is smaller as the current one, update the distance and set the current vertex as antecessor. This step is also called update and this is Dijkstra's central idea. 


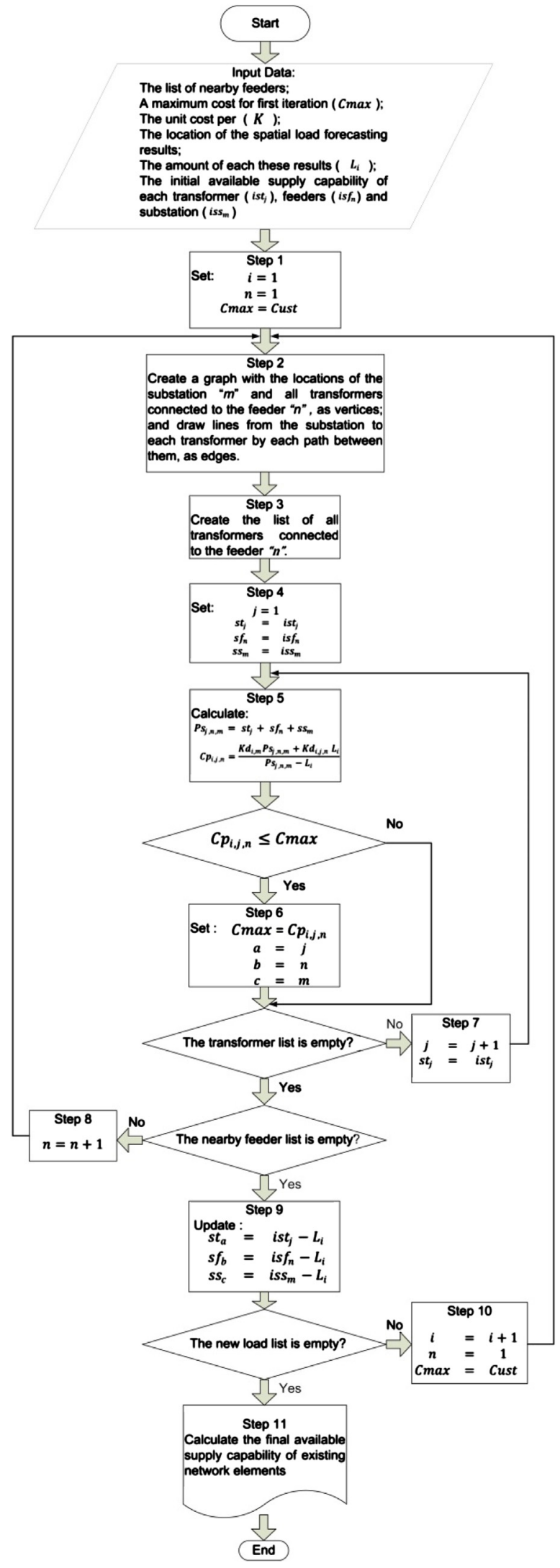

Fig. 2. Flowchart of the proposed method.

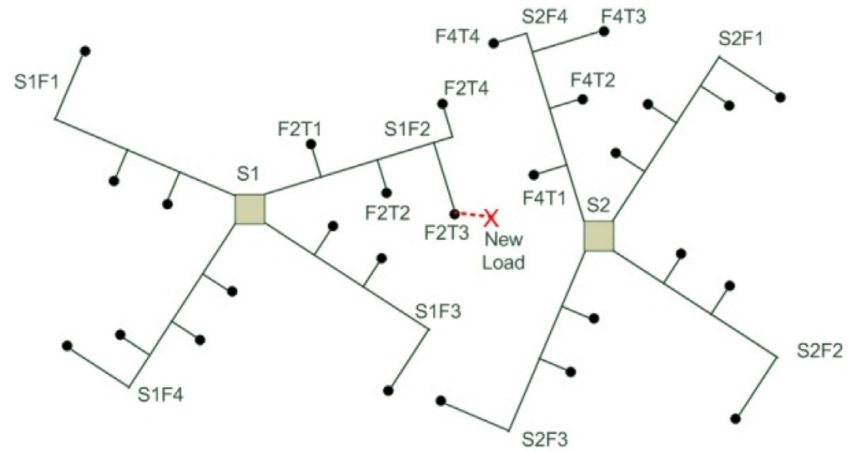

Fig. 3. The distribution system for the allocation example. (For interpretation of the references to color in the text, the reader is referred to the web version of this article.)

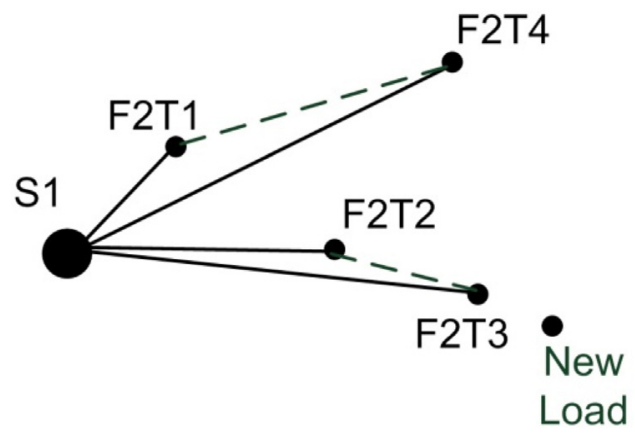

Fig. 4. The graph of the first feeder for the allocation example.

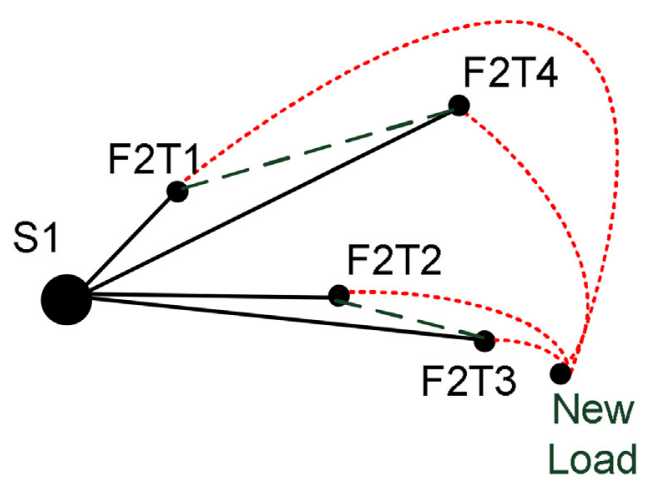

Fig. 5. A possible path to connect the first load in the allocation example using the proposed method. (For interpretation of the references to color in the text, the reader is referred to the web version of this article.)

Step 6-Set the vertex with the minimal temporary distance as active. Mark its distance as permanent.

Repeat steps 1-6 until there is no any new load center marked with a temporary distance. Fig. 1 shows the algorithm for the Dijkstra algorithm.

Dijkstra algorithm can find a good solution, if the cost function is positive. Otherwise, for negative values, must use the Bellman-Ford algorithm; however, such methodology has a higher computational effort than Dijkstra algorithm, as explained in Ref. [15]. Another restriction of the Dijkstra technique for allocating new load is that the weights of the edges (the path's cost function) are invariant during the allocation process. This function cannot upgrade the capacity of the distribution network elements for each load allocated. In order to overcome these restrictions, in the following section is to present the methodology proposed to carry out such allocation. 


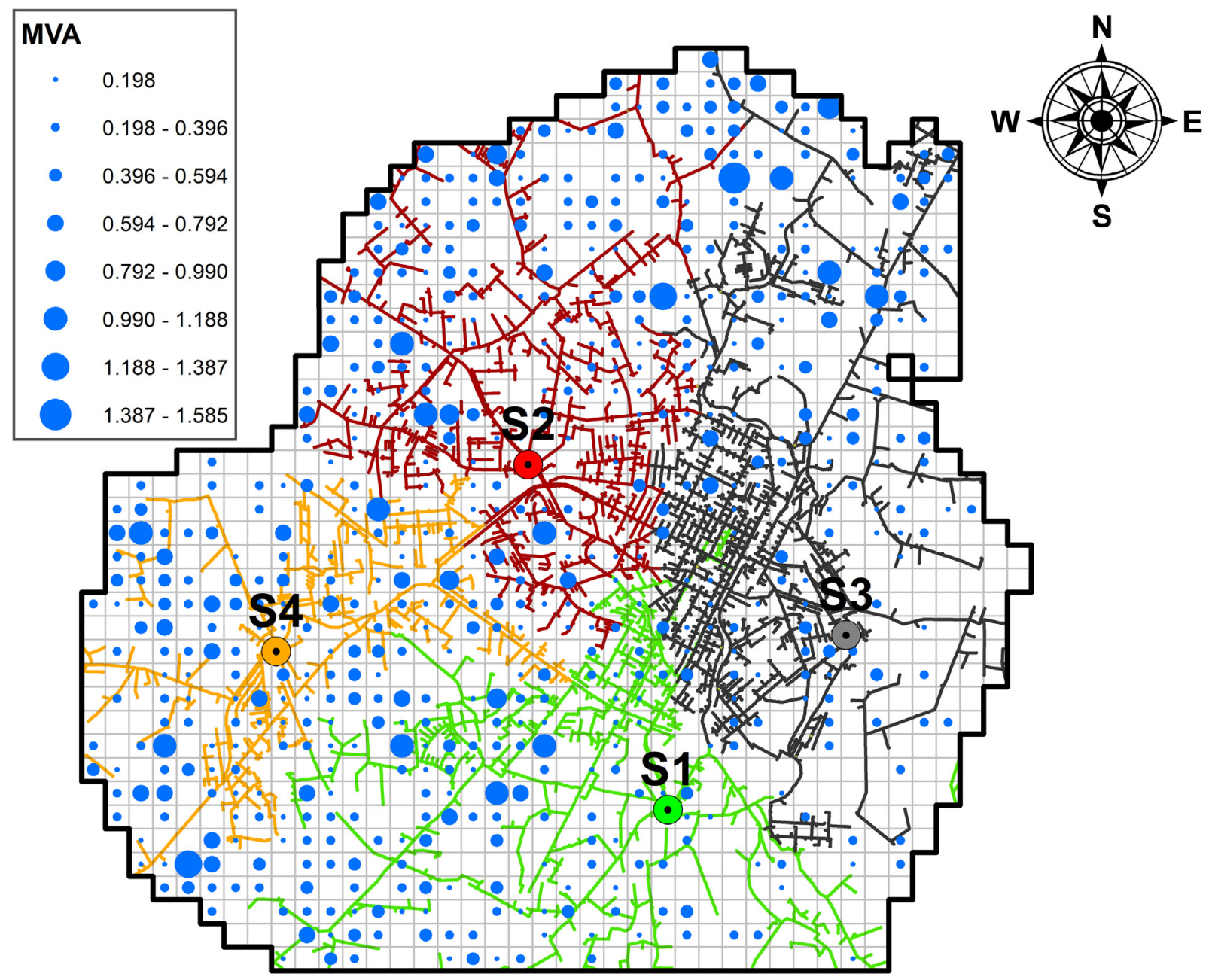

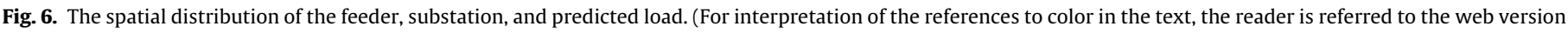
of this article.)

\section{The proposed local search algorithm to allocate loads predicted by spatial load forecasting studies}

The proposed method treats each forecast load as a new load center to be connected by the shortest path to a distribution feeder, searching through a list of feeders near the new load center. To find this path, a graph representing the location of transformers (vertices) and the paths to substations (edges) is constructed. This graph is a representation of the feeder's connections, i.e., the substation and distribution transformers that are connected to it. This graph is a form to represent the search space [15]. The shortest path consists of an edge of the graph and a new line that will be added to connect the new load center. The allocation depends on the path's cost function, which takes into account the capability of the existing distribution network to supply the new loads, seeking to allocate these loads to network elements with higher supply capability. Therefore, the proposed method performs a search in the feeders list of the path with higher capability for each new load center that is allocated, i.e., it finds the path with minimal cost function for list of nearby feeders. After allocate each load, the capability of the network elements are updated, so when the proposal seeks to allocate the next load center, will find a new path with the highest available capacity, this strategy allows to reduce one lower number of overloaded existing network elements (after the allocation process). The allocation performed by the proposed methodology does not attempt to determine the optimal path or route for con- necting each new load, but rather seeks to determine the capability of existing networks to supply the expected loads in the geographic area, in order to support decisions on how to expand the distribution system. The results of the proposal could help to the planners in the creation of a suitable load data for optimal expansion models of the distribution system, reducing the search space of the network elements to be expanded and the cost of the expansion distribution system, as explained in Ref. [9].

\subsection{Input data}

To implement the proposed method, all information must be georeferenced, such as: the location of the forecast loads (the new load centers), the list of nearby feeders for each of these loads, the spatial distribution of the existing network elements and the amount of MVA that each of these elements can supply in addition to their existing loads, which is known as available supply capacity (ASC).

The ASC for each distribution network element is calculated as the power difference, in MVA, between its power supply capacity and the load connected to it. This difference is an extension of the indicator described in Ref. [20], which uses an ASC value for the distribution system as a whole. This indicator is used to determine the capacity to supply part of the load growth before performing the expansion of the distribution system and it can reduce the cost of future installations for the distribution system, as explained in 


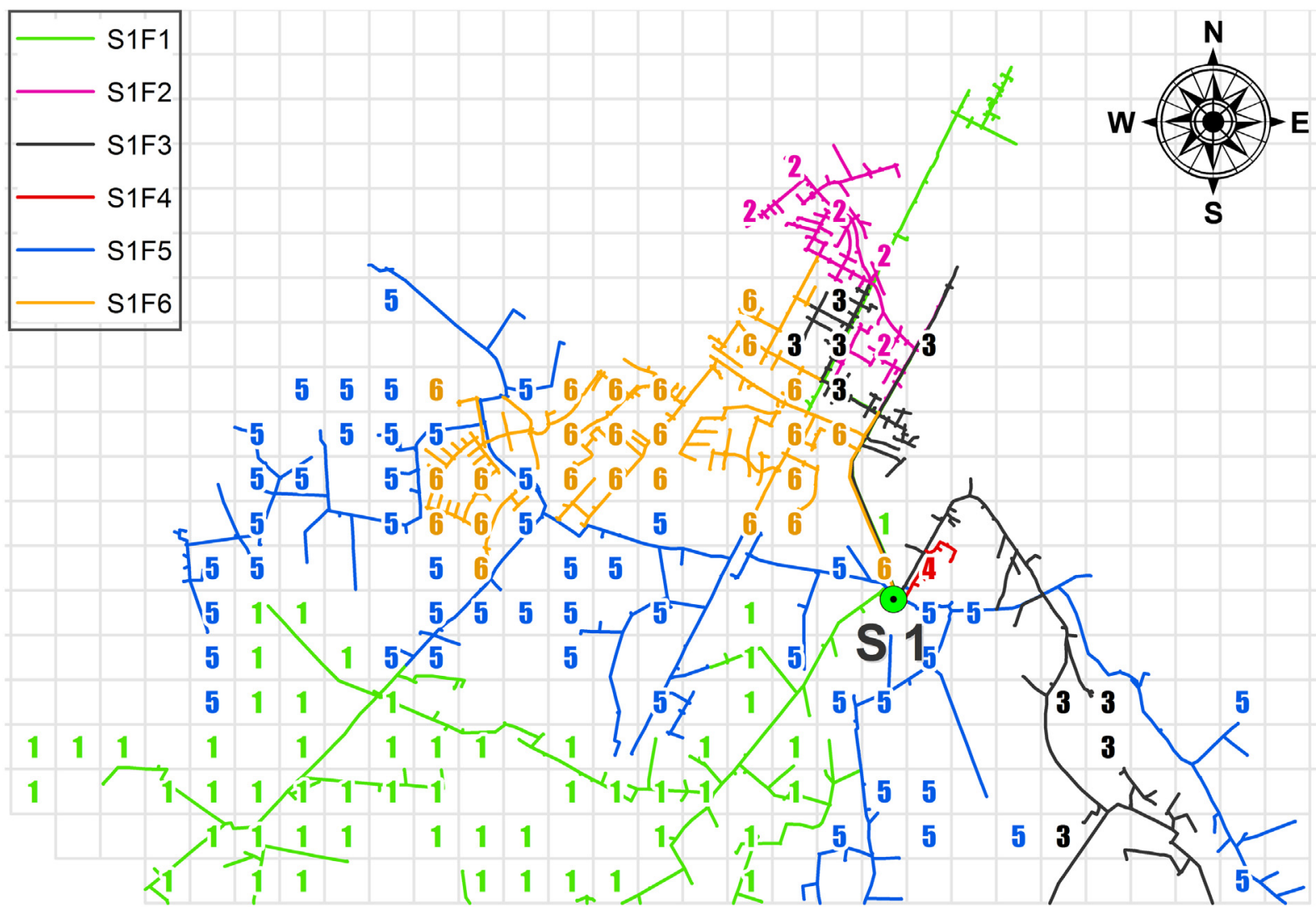

Fig. 7. The load allocation for all feeders of substation S1 by ArcGIS.

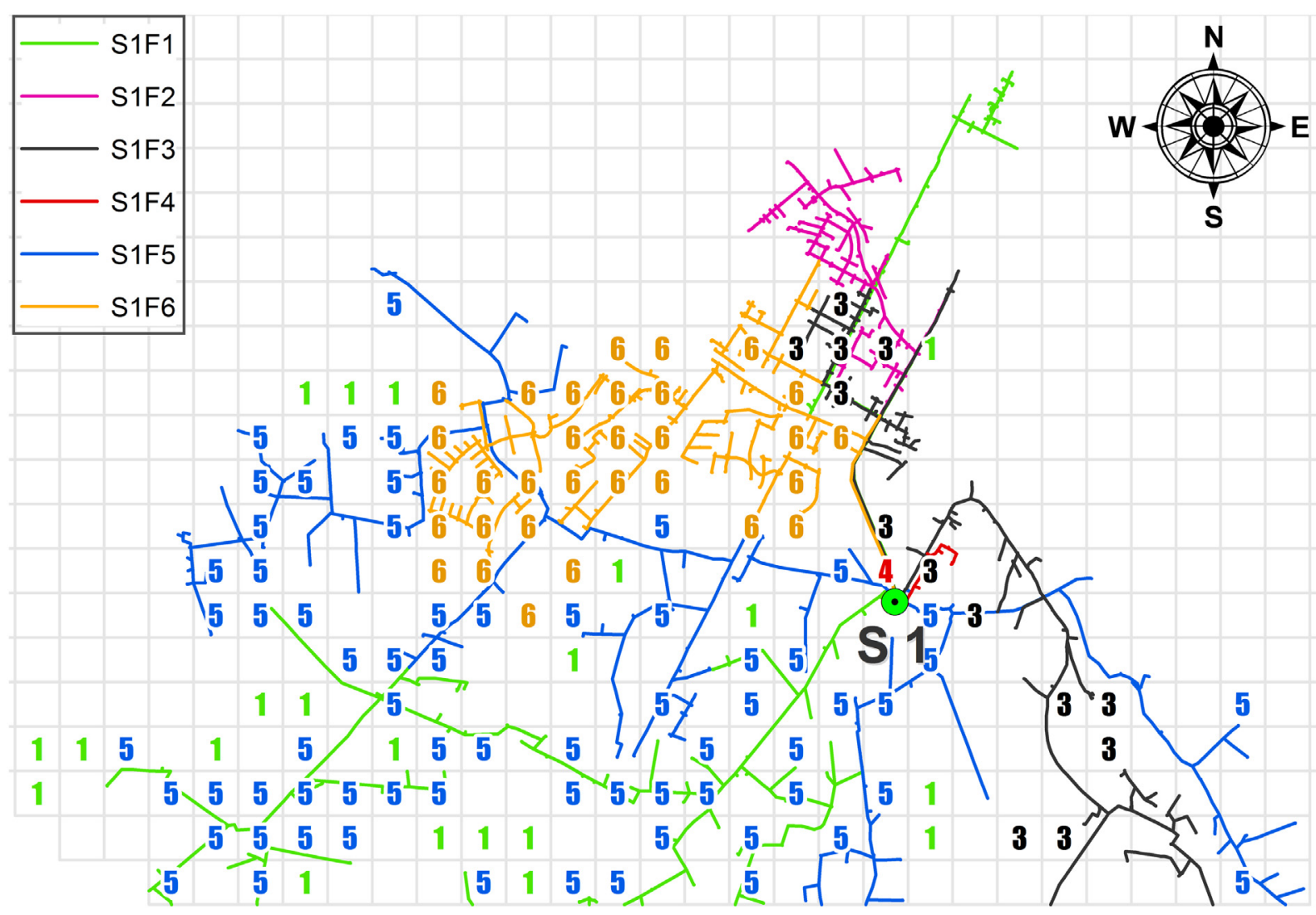

Fig. 8. The load allocation for all feeders of substation S1 using the proposed method. 


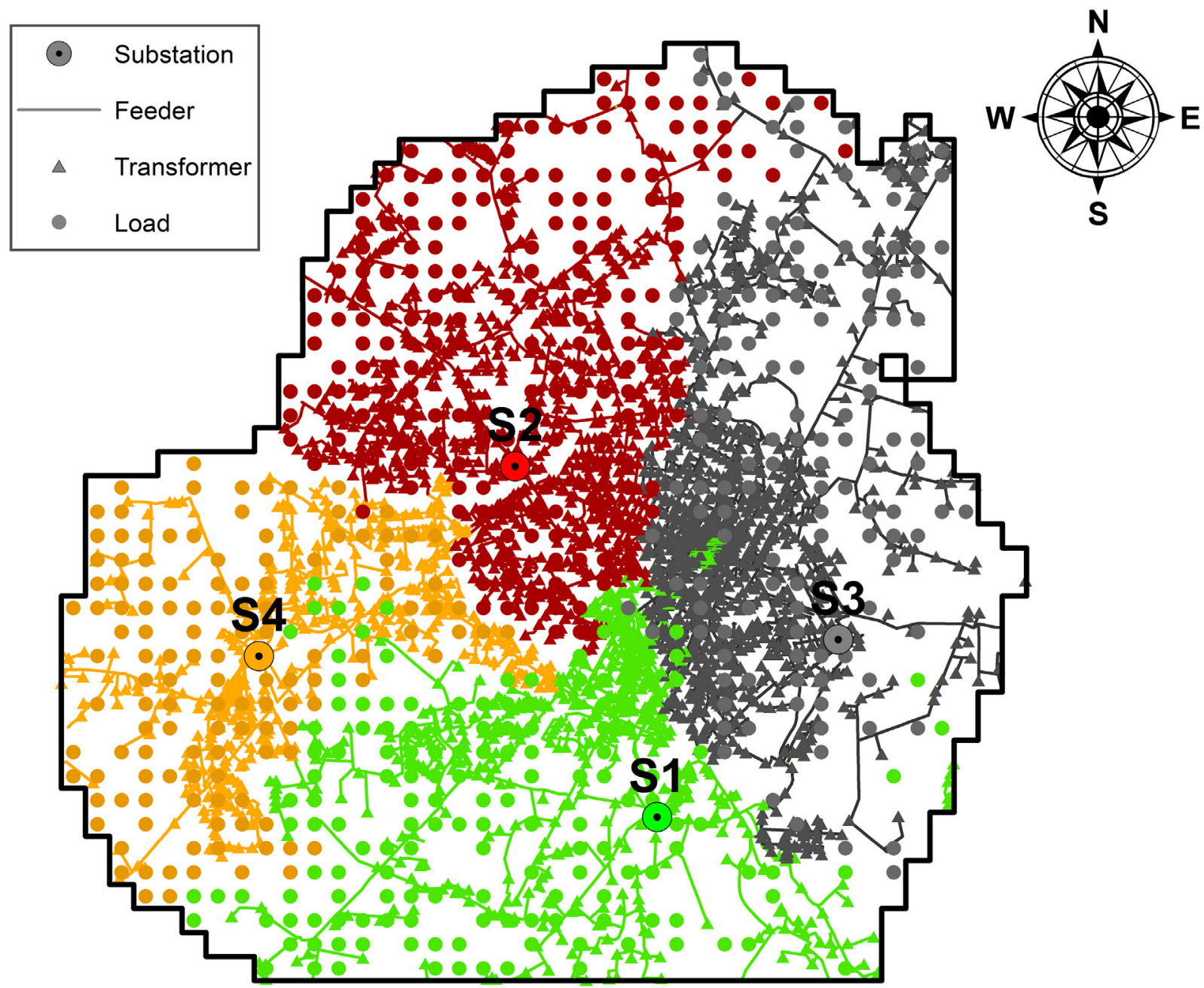

Fig. 9. The substation areas determined using the proposed method.

Ref. [20]. In the specialized literature, the ASC has been used in several studies of the planning of electrical networks, as shown in Ref. [21].

\subsection{List of nearby feeders and new load centers}

The new load centers list consists of the location at which the load of a subarea is concentrated and their respective magnitudes calculated using spatial forecasting methods. This list is ordered from lowest to highest load, in order to assign those with lower loads first. This pre-sorting helps reduce the computational time of local search algorithms [18]. Also, after several experiments with loads from real distribution systems, we found that this sorting helps to reduce the number of overloaded feeders at the end of the load allocation process.

The list containing the nearby feeders for each new load center serves to reduce the search space when assigning a load to a feeder. Some criteria and planner experience can be used to limit the size of the list, such as: defining the maximum distance between a feeder and a new load and the maximum number of nearby feeders. This list will allow diversity in the search for new solutions, which is used to find solutions using metaheuristic techniques [19].

\subsection{The cost of a path}

Ref. [15] explained that the restrictions of the search algorithms to find good solutions depend on proper function of cost. After performing several experiments considering information from real distribution systems, it was found that the path's cost function for each new load center $i$ can be calculated via Eq. (1):

$C p_{i, j, n}=\frac{K d_{i, m} P s_{j, n, m}+K d_{i, j, n} L_{i}}{P s_{j, n, m}-L_{i}}$

where $L_{i}$ is the amount of MVA to connect; $C p_{i, j, n}$ is the cost function for the path connecting load center $i$ to distribution transformer $j$ and feeder $n$ from the candidate list; $K$ is the unit cost per kilometer $(\mathrm{km}) ; d_{i, j, n}$ is the Euclidian distance between load center $i$ and distribution transformer $j$ in $\mathrm{km} ; d_{i, m}$ is the Euclidian distance along the path formed by load center $i$ and substation $m$ in $\mathrm{km}$, and $P s_{j, n, m}$ is the ASC value of the path formed by distribution transformer $j$ in feeder $n$ from substation $m$ in MVA, which is calculated via Eq. (2):

$P s_{j, n, m}=s t_{j}+s f_{n}+s s_{m}$

where $s t_{j}$ is the ASC value of distribution transformer $j$ in $M V A$; $s f_{n}$ is the ASC value of distribution feeder $n$ in $M V A$; and $s s_{m}$ is the ASC value of substation $m$ in $M V A$.

The value of $K$ in expression ( 1 ) is used to improve local solutions during the allocation process, avoiding those that are economically infeasible. The value of $K d_{i, m} P s_{j, n, m}$ is the sum of the costs for the edge traveled, from a new load center to a substation, and $K d_{i, j, n}$ $L_{i}$ is the cost of the new edge that must be built to connect the new load center. This value can be determined from the cost of the distribution feeder, per kilometer. 
a

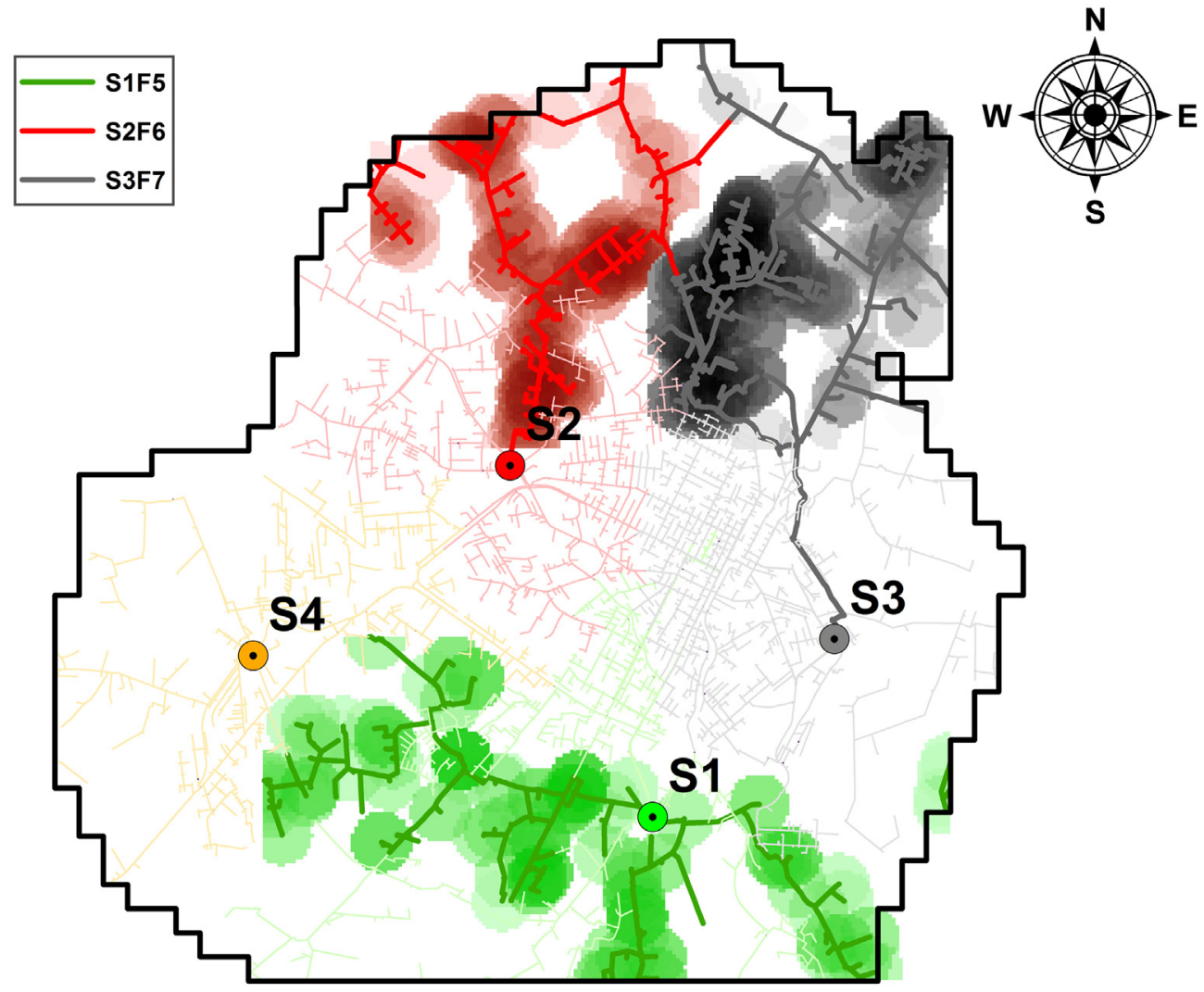

b

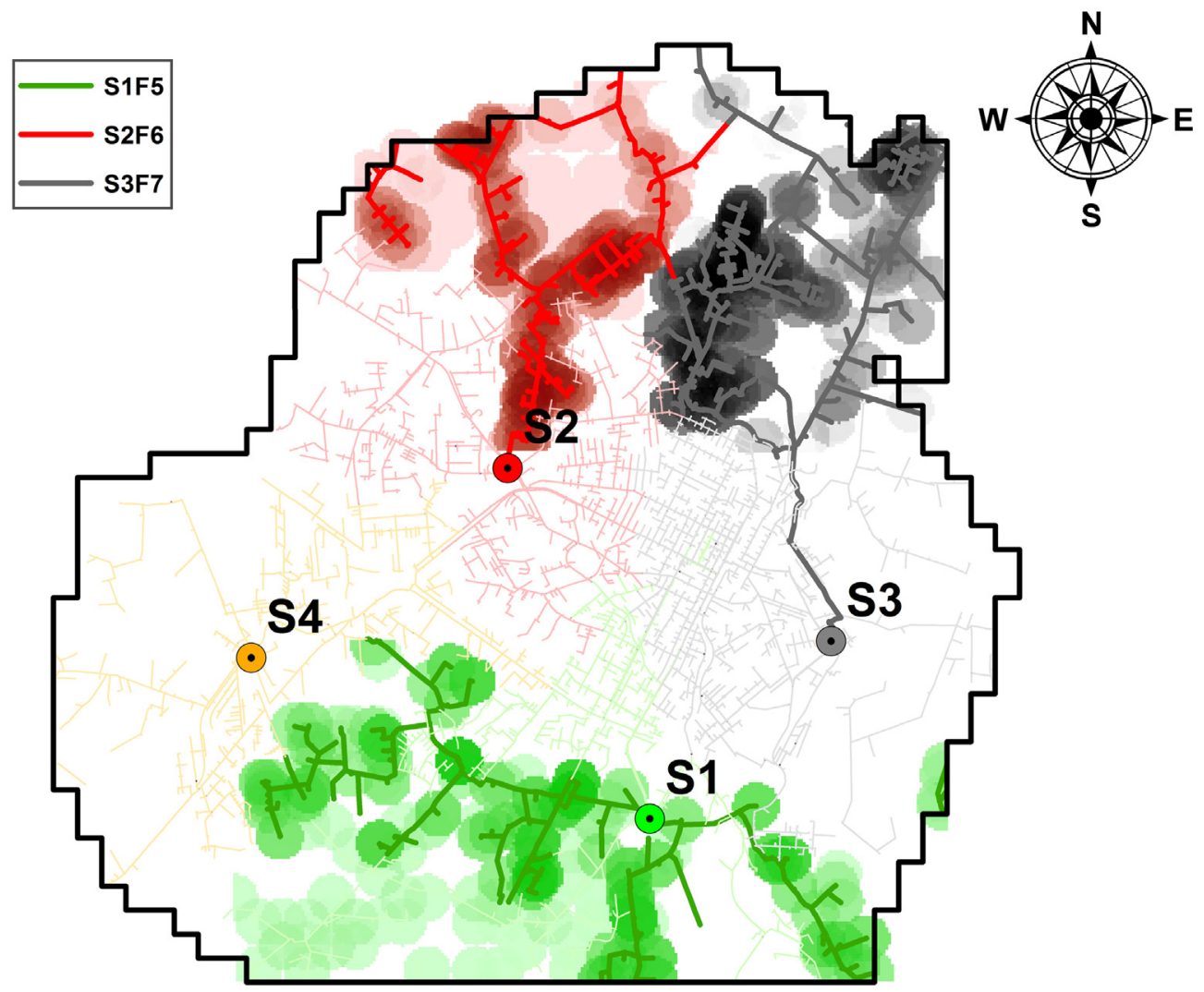

Fig. 10. (a) Initial service area of feeders S1F5, S2F6 and S3F7; (b) final service area of the aforementioned feeders. 
In the allocation process, the value of the numerator in expression (1) increases for paths with higher overhead, while the value of the denominator decreases for paths formed by elements with higher available supply capability; thus, this process prioritizes allocating loads to paths with less overload and greater supply capability, with the lowest $C p_{i, j, n}$, determining the distribution transformer $j$ in feeder $n$ that should be linked to new load center $i$. After allocation of this load, the values of $s t_{j}, s f_{n}$ and $s s_{m}$ are updated. Thus, the cost function is calculated by taking into account the variation in these supply capabilities, which decrease whenever a new load center is linked. The path's cost function is the term used in search algorithms; this function is similar to the objective function in optimization techniques. Search algorithms are known in the optimization area as techniques to solve the shortest path problem, as explained in Ref. [22].

Planners should limit the initial value of $s t_{j}, s f_{n}$ and $s s_{m}$ according to the reliability criteria of the distribution system $[7,23]$. Thus, the supply capability of the distribution networks given as input data is a percentage of the ASC. Techniques and recommendations for calculating the ASC are explained in Refs. [20,21].

The form of calculate the path's cost function presented in this section is a differential of the proposed model. Considering the demonstrated shown in Ref. [15] to solve the shortest path problem, the found solution is the best solution for the search space defined by the planner. It is noted that the proposed model does not seek to optimal solutions to the expansion of distribution system. The proposal can help to reduce the number of candidates of network elements to expand and their cost.

\subsection{Proposed algorithm}

Fig. 2 shows the algorithm for the proposed method. The input data is the list of nearby feeders; a maximum cost for the first iteration (Cust); the unit cost per $\mathrm{km}(K)$; the location of the forecast loads and their magnitude $\left(L_{i}\right)$ in MVA; the path connecting each substation $m$ and transformer $j$; the initial available supply capability of each transformer $j\left(\right.$ ist $\left._{j}\right)$, feeder $n\left(i s f_{n}\right.$ ) and substation $m$ $\left(\right.$ iss $\left._{m}\right)$ and their spatial distribution in the service zone.

As explained in Section 4.2, each new load center is associated with a list of nearby feeders. In Fig. 2, the variable $i$ represents the position of the load center to be connected in the list of new loads. The position of each of the feeders near it is represented by the variable $n$. For each feeder $n$ in the nearby feeder list, the proposed method creates a graph, identifying the substation $m$ that is connected to feeder $n$ and the all distribution transformers linked to this feeder, in order to determine the sum of the costs of the edges traversed from a new load center to a substation. This is equal to $\frac{K d_{i, m} P s_{j, n, m}}{P s_{j, n, m}-L_{i}}$. Our proposed algorithm analyzes the path cost of the new edge that must be built to connect the new load center - equal to $\frac{K d_{i, j, n} L_{i}}{P s_{j, n, m}-L_{i}}-$ in order to obtain the lowest cost path for connecting a new load via expression (1). When the path with the lowest cost is found, it connects the load along this path, and then the ASC value is updated for all path elements as shown in step 9 of Fig. 2.

The proposed algorithm stops when all load centers have been connected to distribution feeders in the service zone (stop criterion). When this criterion is met, the final available supply capability (after the allocation process) of the existing network to supply the expected loads in the geographic area is calculated.

To better understand the proposed algorithm, one allocation in one hypothetical distribution system is demonstrated. This system has two distribution substations, each with four feeders. Fig. 3 shows the spatial distribution of the network elements; the transformers are in black, the substations are in gray, the new load center to allocate is in red, and feeders are black lines. For this allocation, we assume that the maximum cost for the first iteration is 10,000 ,
Table 1

The initial available supply capabilities of the distribution network elements for the allocation example.

\begin{tabular}{ll}
\hline $\begin{array}{l}\text { Distribution } \\
\text { network element }\end{array}$ & $\begin{array}{l}\text { Initial supply } \\
\text { capability (kVA) }\end{array}$ \\
\hline S1 & 150 \\
S2 & 40 \\
S1F1 & 40 \\
S1F2 & 60 \\
S1F3 & 10 \\
S1F4 & 10 \\
S2F1 & 5 \\
S2F2 & 5 \\
S2F3 & 10 \\
S2F4 & 15 \\
\hline
\end{tabular}

Table 2

The new available supply capabilities of the distribution network elements after connection to a load in the allocation example.

\begin{tabular}{ll}
\hline $\begin{array}{l}\text { Distribution } \\
\text { network element }\end{array}$ & $\begin{array}{l}\text { New supply } \\
\text { capability (MVA) }\end{array}$ \\
\hline S1 & 145 \\
S1F2 & 55 \\
F2T3 & 5 \\
\hline
\end{tabular}

the unit cost is $\$ 610$ per kilometer, the load center is $5 \mathrm{MVA}$, and the initial available supply capability of all transformers is 0.1 MVA. The other initial available supply capabilities are shown in Table 1.

In the following, the eleven steps required for one allocation are explained in detail:

Step 1: The new load centers list contains one item. For this new load, the nearby feeders list consists of S1F2 and S2F4. In this step, the counters for load centers and nearby feeders is initialized $(i=1$ and $n=1$, respectively). The value of the path cost variable (Cust) is assigned to the maximum cost for the first iteration ( Cust $=10,000)$.

Step 2: To represent the search space solutions in this step, a graph for the feeder S1F2 is created, with vertices at the locations of substation " $\mathrm{m}$ " and all transformers connected to feeder S1F2. The edges are the existing connections between substation " $\mathrm{m}$ " and these transformers, as shown in Fig. 4. In this figure, the dashed lines are possible paths that can be used to close the normally open switches. Thus, the proposed method can work with radial or interconnected networks. The substation connected to feeder S1F2 is S1; so, the substation identifier $(m)$ has the value of $1(m=1)$.

Step 3: There are four transformers connected to feeder S1F2; so the transformers list has four elements.

Step 4: Initialize the transformer counter $(j=1)$.

Step 5-7: These steps are executed until the transformer list is empty. In each pass, the method calculates the path cost via expressions (1) and (2), saving the lower value. Each possible path to connect the new load center is shown using red dashed lines in Fig. 5. The lowest cost is $\$ 871.18$ for transformer F2T3.

Step 8: This step is executed because the nearby feeders list is not empty, so the method increments the feeder counter (to $n=2$ ) and returns to step 2 .

Step 2-7: These steps are executed until the transformer list for feeder S2F4 is empty. The substation connected to this feeder is $\mathrm{S} 2$, so $\mathrm{m}=2$. In each pass, the method calculates the path cost via expressions (1) and (2), comparing each calculated cost with the lowest value, which was found for feeder S1F2. These costs are higher than $\$ 871.18$, so the load center is connected to feeder S1F2.

Step 9: In this step the supply capability of the transformers, feeder and substation are updated, with the results shown in Table 2.

Step 10: is executed if there are further load centers to connect. In this step, the load center counter is incremented $(i=i+1)$, the nearby feeder counter is initialized $(n=1)$ and the value of the path 
Table 3

The final available supply capabilities of the distribution network elements in the allocation example.

\begin{tabular}{lll}
\hline $\begin{array}{l}\text { Distribution } \\
\text { Network Element }\end{array}$ & $\begin{array}{l}\text { Initial Supply } \\
\text { Capability (MVA) }\end{array}$ & $\begin{array}{l}\text { Initial Supply } \\
\text { Capability (MVA) }\end{array}$ \\
\hline S1 & 150 & 145 \\
S2 & 40 & 40 \\
S1F1 & 40 & 40 \\
S1F2 & 60 & 55 \\
S1F3 & 10 & 10 \\
S1F4 & 10 & 10 \\
S2F1 & 5 & 5 \\
S2F2 & 5 & 5 \\
S2F3 & 10 & 10 \\
S2F4 & 15 & 15 \\
\hline
\end{tabular}

cost variable (Cust) is assigned the maximum cost (Cust $=10,000)$. For this example, this step is not performed.

Step 11: The load centers list is empty, and the proposed method calculates the new available capability of the existing network elements, shown in Table 3.

\section{Results}

The proposed method was applied to a medium-sized city in Brazil with four distribution substations and thirty feeders. The input data was extracted from Ref. [24], and is grouped into grid cells on a regular grid.

Application of the method requires the location of new load centers (georeferenced data). These locations can be obtained from a geographic information system (GIS). To do this, we used the free software TerraView [25] to obtain the geographic coordinates for the center of each grid cell in the area studied in spatial load forecasting.

Fig. 6 shows the spatial distribution of the distribution feeders installed in the area studied and the expected total load centers for a five-year horizon, estimated using the simulated model presented in Ref. [24]. The load center is represented by blue dots in the center of each grid cell. The spatial resolution is $0.12 \mathrm{~km}^{2}$ (the area of each grid cell is $0.34 \mathrm{~km} \times 0.34 \mathrm{~km}$ ), forming a $40 \times 40$ grid ( 1600 cells). The period of five years was used due to a law imposed on cities in Brazil regarding the expansion of feeder networks [26]. The estimated load growth for during the five-year horizon period is $239.56 \mathrm{MVA}$, which represents of the installed load by about $18 \%$, with increased load in 600 new load centers.

When applying the proposed method, each of the geographic coordinates corresponding to the center of a grid cell is associated with distribution feeders. The distribution feeder cost per kilometer $(K)$ is $\$ 100,000$, which is the approximate cost for installation of new low-voltage feeders in the service area. Also, the maximum cost of the first iteration is $1,000,000$. The initial supply capacity of the distribution substation and feeder are shown in Table 4.

In order to show the effectiveness of the proposed method, the results of the allocation are compared with the association performed by the allocation-location model available in ArcGis [11]. This model uses a Dijkstra algorithm, which is initialized with the ASC values of each feeder as edge costs. These costs are not changed by the Dijkstra algorithm during the allocation process, as shown in Section 3. In this comparison, we tested two lists of nearby feeders; one list with a nearby feeder and the other with two nearby feeders for the center point of each grid. These lists were constructed by identifying the feeders crossing the square cells in Fig. 6.

The overload and final available supply capability of each feeder and substation are shown in Table 5, for both the proposed method and ArcGIS. We observed that the total overload and ASC of the distribution system when using the proposed method are similar to the values found using ArcGIS. However, the solution found using
Table 4

The initial available supply capabilities of the distribution network elements for application of the proposed method.

\begin{tabular}{ll}
\hline Distribution & $\begin{array}{l}\text { Initial supply } \\
\text { capability (MVA) }\end{array}$ \\
network element & 108.53 \\
\hline S1 & 209.56 \\
S2 & 873.00 \\
S3 & 131.61 \\
S4 & 16.11 \\
S1F1 & 16.10 \\
S1F2 & 16.82 \\
S1F3 & 19.90 \\
S1F4 & 16.11 \\
S1F5 & 15.71 \\
S1F6 & 14.19 \\
S2F1 & 18.21 \\
S2F2 & 16.60 \\
S2F3 & 18.54 \\
S2F4 & 16.49 \\
S2F5 & 15.58 \\
S2F6 & 14.68 \\
S2F7 & 19.89 \\
S2F8 & 16.26 \\
S2F9 & 16.10 \\
S3F1 & 15.57 \\
S3F2 & 17.20 \\
S3F3 & 14.44 \\
S3F4 & 15.38 \\
S3F5 & 13.37 \\
S3F6 & 14.95 \\
S3F7 & 16.11 \\
S3F8 & 16.10 \\
S4F9 & 15.54 \\
S4F2 & 19.61 \\
S4F3 & 19.61 \\
S4F4 & 15.79 \\
S4F5 & 19.99 \\
S4F6 & 17.85 \\
\hline &
\end{tabular}

the proposed algorithm has a lower number of overloaded distribution networks elements than the solution found using the Dijkstra method. This is important as it reduces the number of facilities to be expanded and their cost [23].

The cost to expand distribution to the most overloaded feeders would be $\$ 6,732,504$ using the solution found by ArcGIS, and $\$ 5,914,112$, using the solution found using proposed method. These costs are different because the proposed methodology takes into account the ASC values of the distribution transformers, seeking to reduce transformer overload. The model available in ArcGIS does not take these values into account during the allocation process. The cost avoided by expanding the network elements was not shown, because of be outside the scope of this work. However, based on the case studies presented in Ref. [9], it is expected that distribution utilities can avoid the construction and installation of new facilities in its distribution system.

Table 5 identifies the overloaded electrical network elements and how much the supply capability in each of these elements must be changed, providing useful information that planners can use when making distribution planning decisions.

A nearby feeder list with a high number of feeders can be constructed, but using this list can result in unviable solutions in financial terms because of increased path costs to new loads. If the planner needs to use a list with a greater number of nearby feeders, then an appropriate cost per $\mathrm{km}$ should be used.

Figs. 7 and 8 show the load allocation for all feeders of substation S1 calculated using ArcGIS and the proposed method. Each load is indicated by a number from one to six, representing the feeder to which it was connected. Note that the main difference is in the number of loads allocated to the S1F1 and S1F5 feeders. The proposed method allocated more loads to the S1F5 feeder than 
Table 5

The overload and final supply capability of the distribution network elements for application of the proposed method.

\begin{tabular}{|c|c|c|c|c|c|c|}
\hline \multirow[t]{2}{*}{ Distribution network element } & \multicolumn{2}{|l|}{ ArcGIS } & \multicolumn{2}{|c|}{ The proposed method with 1 nearby feeder } & \multicolumn{2}{|c|}{ The proposed method with 2 nearby feeders } \\
\hline & $\begin{array}{l}\text { Final supply } \\
\text { capability (MVA) }\end{array}$ & $\begin{array}{l}\text { Overload } \\
\text { (MVA) }\end{array}$ & $\begin{array}{l}\text { Final supply } \\
\text { capability (MVA) }\end{array}$ & $\begin{array}{l}\text { Overload } \\
\text { (MVA) }\end{array}$ & $\begin{array}{l}\text { Final supply } \\
\text { capability (MVA) }\end{array}$ & $\begin{array}{l}\text { Overload } \\
\text { (MVA) }\end{array}$ \\
\hline S1 & 54.44 & 0.00 & 55.63 & 0.00 & 49.68 & 0.00 \\
\hline S2 & 141.59 & 0.00 & 141.20 & 0.00 & 132.88 & 0.00 \\
\hline S3 & 814.35 & 0.00 & 812.56 & 0.00 & 822.67 & 0.00 \\
\hline S4 & 72.76 & 0.00 & 73.75 & 0.00 & 77.92 & 0.00 \\
\hline S1F1 & 0.00 & 3.90 & 4.61 & 0.00 & 0.06 & 0.00 \\
\hline S1F2 & 14.51 & 0.00 & 16.10 & 0.00 & 16.10 & 0.00 \\
\hline S1F3 & 13.85 & 0.00 & 12.66 & 0.00 & 12.26 & 0.00 \\
\hline S1F4 & 19.31 & 0.00 & 19.70 & 0.00 & 19.70 & 0.00 \\
\hline S1F5 & 0.00 & 3.90 & 0.00 & 8.66 & 0.00 & 8.86 \\
\hline S1F6 & 6.79 & 0.00 & 3.42 & 0.00 & 2.63 & 0.00 \\
\hline S2F1 & 8.25 & 0.00 & 8.84 & 0.00 & 8.84 & 0.00 \\
\hline S2F2 & 15.04 & 0.00 & 14.64 & 0.00 & 14.64 & 0.00 \\
\hline S2F3 & 15.81 & 0.00 & 14.82 & 0.00 & 14.82 & 0.00 \\
\hline S2F4 & 17.55 & 0.00 & 17.55 & 0.00 & 17.55 & 0.00 \\
\hline S2F5 & 4.80 & 0.00 & 7.17 & 0.00 & 7.17 & 0.00 \\
\hline S2F6 & 0.00 & 14.54 & 0.00 & 13.75 & 0.00 & 22.86 \\
\hline S2F7 & 4.38 & 0.00 & 1.60 & 0.00 & 1.60 & 0.00 \\
\hline S2F8 & 19.30 & 0.00 & 19.30 & 0.00 & 19.30 & 0.00 \\
\hline S2F9 & 11.90 & 0.00 & 11.90 & 0.00 & 12.70 & 0.00 \\
\hline S3F1 & 13.52 & 0.00 & 14.71 & 0.00 & 14.71 & 0.00 \\
\hline S3F2 & 14.58 & 0.00 & 14.98 & 0.00 & 14.98 & 0.00 \\
\hline S3F3 & 16.41 & 0.00 & 15.41 & 0.00 & 15.41 & 0.00 \\
\hline S3F4 & 10.87 & 0.00 & 12.06 & 0.00 & 12.65 & 0.00 \\
\hline S3F5 & 10.82 & 0.00 & 11.22 & 0.00 & 11.62 & 0.00 \\
\hline S3F6 & 5.25 & 0.00 & 4.65 & 0.00 & 4.65 & 0.00 \\
\hline S3F7 & 0.00 & 18.14 & 0.00 & 19.73 & 0.00 & 10.61 \\
\hline S3F8 & 13.34 & 0.00 & 12.94 & 0.00 & 12.94 & 0.00 \\
\hline S3F9 & 13.92 & 0.00 & 12.53 & 0.00 & 12.53 & 0.00 \\
\hline S4F1 & 5.63 & 0.00 & 7.41 & 0.00 & 10.19 & 0.00 \\
\hline S4F2 & 4.35 & 0.00 & 3.56 & 0.00 & 3.56 & 0.00 \\
\hline S4F3 & 13.07 & 0.00 & 9.30 & 0.00 & 9.30 & 0.00 \\
\hline S4F4 & 0.00 & 1.85 & 0.33 & 0.00 & 0.33 & 0.00 \\
\hline S4F5 & 19.59 & 0.00 & 19.79 & 0.00 & 19.79 & 0.00 \\
\hline S4F6 & 8.74 & 0.00 & 10.13 & 0.00 & 11.51 & 0.00 \\
\hline Total overloaded elements & 5.00 & & 3.00 & & 3.00 & \\
\hline Total & 1384.70 & 42.33 & 1384.49 & 42.14 & 1384.68 & 42.34 \\
\hline
\end{tabular}

ArcGIS. Thus, the proposed method overloads this feeder more than the load allocation performed by ArcGIS (see Table 4). However, the sum of available supply capability from all feeders of this substation is higher than the capability determined by ArcGIS for one nearby feeder. This is important because of the lead time for adding new facilities in the distribution system $[8,9]$.

For illustrative purposes, we show the service area of each substation in Fig. 9. This diagram includes the new load centers connected to the distribution feeders. Comparing Figs. 6 and 9, note that the geographic region of substations $\mathrm{S} 3$ and $\mathrm{S} 4$ has grown to the north and west. The substations are not overloaded, but the supply capability of S1 is lower than that of the other substations because the load growth for S1 is the highest for the five-year period. This information is important to the planner, who will need to decide whether to build a new substation in this region or reinforce substation S1.

Ref. [6] explained the importance of dividing the study area into several subareas, considering the area service of distribution network elements, in order to obtain information for the expansion planning of distribution systems. Based on this information, the planners can decide whether to reinforce and rearrange the existing distribution network or build new feeders in some regions. The make-decisions for expansion planning are outside the scope of this paper. The proposed methodology can help in determining the expansion of such subareas. Fig. 10 shows the initial and final service area of the feeders S1F5, S2F6 and S3F7, which are the most overloaded feeders, according to the configuration found by the proposed algorithm. This area was created using the Kernel maps tool available in ArcGIS. This map helps to planners visualize the concentration of load centers within the area studied. This figure shows the importance of the proposed method, which draws the attention of planners to the expansion of the feeder service area, being necessary to analyze the possibility to install electrical facilities in the expanded urban zones.

Although the proposal to perform a cost analysis of all feeders entered as input data, the computational time to perform these calculations is similar to the time used for the construction of graphical representation necessaries in commercial software. The proposal makes a reduction in the time of graphics, only considering that new load centers can be connected to a nearby transformer; the proposal can be performed with reduction of time of graphic constructions. The computational time to allocate the forecast load growth using the proposed algorithm is $1.8 \mathrm{~min}$, while the Dijkstra algorithm takes $1.3 \mathrm{~min}$, both using a computer with an Intel Core i5 $1.8 \mathrm{GHz}, 4 \mathrm{~GB}$ RAM memory, using the software $\mathrm{R}$, version 2.13.2 [27]. The proposed method can be implemented in any programming language or in GIS software. Given this computational time, the proposed algorithm found a solution with a lower number of overloaded elements in a time similar to that of the Dijkstra algorithm available in GIS software.

\section{Conclusions}

The current scenario of remodeling distribution networks together with redevelopment of urban areas requires that planners make decisions in order to meet the demands of load growth, while identifying capacity shortfalls in power network elements and deciding where to locate new facilities. For direct expansion 
of the distribution network, distribution utilities need access to the results of spatial forecasting, performed using GIS and other techniques, displaying the expected spatial load distribution on thematic maps. In order for these maps to aid distribution system design, the forecast loads must be allocated to distribution network elements. In this context, a shortest path method that allocates the loads predicted using spatial load forecasting whilst seeking to avoid overloads in existing network elements was presented. The method determines the final available capability of existing networks to provide sufficient power to supply the forecast load, providing information that helps planners when making decisions on how to expand the distribution system.

The proposed method and Dijkstra algorithm are similar in terms of computational time and the ability to work with a large amount of input data. In the application of the method, the computational time to allocate the 1600 load centers is $0.5 \mathrm{~min}(30 \mathrm{~s})$ higher than Dijkstra algorithm. However, the proposed method can work with positive and negative values of the path's cost function, being this a differential of the proposal in comparison of search algorithms that consider these values, but with a higher computational effort.

One contribution of this method is to take into account the variation in the available capability of existing networks to supply the expected loads during the allocation process via an appropriate the path's cost function. This function allows obtaining a lower number of overloaded existing distribution network elements than similar methods available in GIS software. Table 5 shows that in the application, the proposal found a solution with two overloaded elements less than the Dijkstra algorithm.

The output of the algorithm is the final available capability of the existing network elements to supply the forecast load. This information is extremely valuable for decisions regarding investments needed to expand power networks. In the application of the propose algorithm, the cost to expand distribution to the most overloaded feeders is $\$ 818,392$ less than the solution found by ArcGIS. This difference decreases investments in planning expansion of the distribution system.

\section{Acknowledgments}

The authors gratefully acknowledge the contributions of the CENTROSUR distribution utility to their work on the original version of this document; and CNPq and FAPESP (Grants $303817 / 2012-7,444743 / 2014-6$, and 2014/06629-0) for their economic support of this project.

\section{References}

[1] C.-W. Ten, E. Wuergler, H.-J. Diehl, Extraction of geospatial topology and graphics for distribution automation framework, IEEE Trans. Power Syst. 23 (November (4)) (2008) 1776-1782.

[2] Y.X. He, et al., Forecasting the urban power load in China based on the risk analysis of land-use change and load density, Int. J. Electr. Power Energy Syst. 73 (2015) 71-79.
[3] J.D. Melo, et al., Grid-based simulation method for spatial electric load forecasting using power-law distribution with fractal exponet, Int. Trans. Electr. Energy Syst. 26 (2016) 1339-1357.

[4] M.-A. Rostami, A. Kavousi-Fard, T. Niknam, Expected cost minimization of smart grids with plug-in hybrid electric vehicles using optimal distribution feeder reconfiguration, IEEE Trans. Ind. Inf. 11 (April (2)) (2015) 388-397.

[5] N. Komninos, E. Philippou, A. Pitsillides, Survey in smart grid and smart home security: issues, challenges and countermeasures, IEEE Commun. Surv. Tutorials 16 (April (4)) (2014) 1933-1954.

[6] H.L. Willis, Spatial Electric Load Forescasting, 2nd ed., Marcel Dekker, New York, 2002.

[7] A.S. Pabla, Electric Power Distribution, McGraw-Hill, New Delhi, 2005.

[8] E.-C. Yeh, H. Tram, Information integration in computerized distribution system planning, IEEE Trans. Power Syst. 12 (May (2)) (1997) 1008-1013.

[9] H.L. Willis, H. Tram, M.V. Engel, L. Finley, Optimization applications to power distribution, IEEE Comput. Appl. Power 8 (October (4)) (1995) 12-17.

[10] P.S. Georgilakis, N.D. Hatziargyriou, A review of power distribution planning in the modern power systems era: models, methods and future research, Electr. Power Syst. Res. 121 (April) (2015) 89-100.

[11] ESRI, ArcGis Resources. Available from: <http://resources.arcgis.com/en/home/> (Accessed 1 May 2015).

[12] S.-U. Rahman, D.K. Smith, Use of location-allocation models in health service development planning in developing nations, Eur. J. Oper. Res. 123 (June (3)) (2000) 437-452.

[13] A. Fredriksson, Location-allocation of public services-citizen access, transparency and measurement. A method and evidence from Brazil and Sweden, Socio-Economic Planning Sciences, to be published, doi:10.1016/j.seps.2016.09.008, [Online] Available from: <www.sciencedirect.com/science/article/pii/S0038012116300015>

[14] J.A. Keane, T.A. Ward, A computational framework for location analysis, IEEE Trans. Syst. Man Cybern. A 32 (September (5)) (2002) 574-581.

[15] B.V. Cherkassky, A.V. Goldberg, T. Radzik, Shortest paths algorithms: theory and experimental evaluation, Math. Program. 73 (May (2)) (1996) 129-174.

[16] J. Shu, et al., A new method for spatial power network planning in complicated environments, IEEE Trans. Power Syst. 27 (1) (2012) 381-389.

[17] M. Held, R.B. Williamson, Creating electrical distribution boundaries usign computational geometry, IEEE Trans. Power Syst. 19 (August (3)) (2014) 1342-1347.

[18] G. Chartrand, P. Zhang, A First Course in Graph Theory, Dover ed., McGraw-Hill Higher Education, Boston, 2012.

[19] F.S. Hillier, G.J. Lieberman, Introduction to Operations Research, 7th ed., McGraw-Hill, New York, 2001.

[20] J. Xiao, et al., Total supply capability and its extended indices for distribution systems: definition, model calculation and applications, IET Gener. Transm. Distrib. 5 (February (8)) (2011) 869-876.

[21] K. Chen, et al., A method to evaluated total supply capability of distribution systems considering network reconfiguration and daily load curvers, IEEE Trans. Power Syst. 31 (May (3)) (2016) 2096-2104.

[22] M.S. Bazaraa, J.J. Jarvis, H.D. Sherali, Linear Programming and Network Flows, 4th ed., Jonh Wiley \& Sons, New Jersey, 2009

[23] T. Gonen, Electric Power Distribution System Engineering, 3rd ed., CRC Press, Boca Raton, 2014.

[24] J.D. Melo, E.M Carreno, A. Padilha-Feltrin, Multi-agent simulation of urban social dynamics for spatial load forecasting, IEEE Trans. Power Syst. 27 (November (4)) (2012) 1870-1878.

[25] TERRAVIEW 4.2.0. TerraView, São Jose dos Campos, January 2010. Available from: <www.dpi.inpe.br/terraview. (In Portuguese)>.

[26] Agência Nacional DE Energia Elétrica-ANEEL, Procedimentos de Distribuição-PRODIST 2008, Procedimentos de Distribuição de Energia Elétrica no Sistema Elétrico Nacional-PRODIST, Abril 2013. Available from: $<$ www.aneel.gov.br/. (In Portuguese)>.

[27] R Development Core TEAM, R: A Language and Environment for Statistical Computing, 2011, Available from: http://www.R-project.org/. 